\title{
High Accuracy Positioning using Jet Thrusters for Quadcopter
}

\author{
ChenHuan $\mathrm{Pi}^{1}$, JinKwan Lin ${ }^{2}$ and Stone Cheng ${ }^{3}$ \\ 1,2,3 National Chiao Tung University, Department of Mechanical Engineering, Taiwan
}

\begin{abstract}
A quadcopter is equipped with four additional jet thrusters on its horizontal plane and vertical to each other in order to improve the maneuverability and positioning accuracy of quadcopter. A dynamic model of the quadcopter with jet thrusters is derived and two controllers are implemented in simulation, one is a dual loop state feedback controller for pose control and another is an auxiliary jet thruster controller for accurate positioning. Step response simulations showed that the jet thruster can control the quadcopter with less overshoot compared to the conventional one. Over 10s loiter simulation with disturbance, the quadcopter with jet thruster decrease $85 \%$ of RMS error of horizontal disturbance compared to a conventional quadcopter with only a dual loop state feedback controller. The jet thruster controller shows the possibility for further accurate in the field of quadcopter positioning.
\end{abstract}

\section{Introduction}

A quadcopter (Quadrotor) is a Micro Aerial Vehicle (MAV) driven by four rotors. The control technique and applications of a quadcopter are popular research topics in recent years due to their low price, small size and easy to modification. Their maneuverability and ease to use make them fit to provide stable flight, navigate into places that ground robots or human cannot reach and perform tasks.

Some applications such as entertainment and search and rescue do not need extremely accurate positioning. However, for interaction with the environment at close range, such as in canopy sampling [1] or factory assemble line delivery, high accuracy positioning is required.

Traditionally, horizontal translation of quadcopter relies on vector component of force changed by tilting the quadcopter a certain angle. Increasing the tilt angle increases lift loss and wind cross-section area. To ameliorate this situation, Bannwarth et al. [2] designed a wind disturbance rejection nonlinear controller on quadcopter. Their experimental results showed that the controller had a position error below 1 meter. Chau and William proposed a continuous robust controller of attitude trajectory tracking for a quadcopter in the presence of uncertainty and unknown disturbance [3]. For these controllers, minimizing the error in the position of the quadcopter under unpredictable circumstances is essential.

In a flight vehicle, many different control methods can be used. The jet system is one of the actuator among these methods and commonly used in flight vehicle attitude control system several years. Jet engines can be classified as air-breathing engines such as turbojet engines and non-air-breathing engines such as rocket engines.

The cold gas thruster is the simplest case in the rocket engines. Unlike other engines used fuel burning, there is no combustion chamber in this system. The exhaust gas was driven by the pressure difference between the tank and outside, not by a chemical reaction. These unbalance pressure push and accelerate the gas flow to drive the system movement. Although this engine cannot supply as much power as fuel burning engines, using for attitude control is enough. [4,5] show the different usage of fuel burning engine from cold gas thruster, which drive quadcopter by gears and transmissions.

This system can be realized by using components such as pressurized gas tank, regulator valve, solenoid and nozzle to create thrust force needed to perform translational or rotational maneuver of vehicle. Many researches provide the feasibility and effectiveness of this system. John G. Furumo [6] demonstrated his system design and cold gas propulsion system that generate 1-D rotation movement of test platform. Assad Anis [7] compared performance values for several cold gas propellants and hardware designs. In [8], a flying vehicle is developed using nitrogen as propellants which is applied in exploration to other celestial bodies.

The rest of this paper presents a control strategy of the combination of quadcopter system and cold gas thruster system. Four cold gas thrusters mounted perpendicular to each other on the quadcopter generate the horizontal force needed to achieve the control goal. However, increasing control inputs in original quadcopter system makes it more complex and more challenge in controller design. Section 2 describes the physical configuration and mathematical system model. Section 3 discusses the 
controller design. Section 4 shows the simulation results. Conclusion and future works are presented in Section 5.

\section{Dynamic model}

\subsection{Jet Thruster Quadcopter}

Unlike the traditional quadcopter in Fig. 1, the jet thruster quadcopter adds four nozzles along the quadcopter arms which can supply additional force on its horizontal plane. These thrusters can be used to improve horizontal maneuverability for more accurate positioning and disturbance rejection.

The model dynamic equations would become Eq. 1,

$$
\left[\begin{array}{c}
m \ddot{x} \\
m \ddot{y} \\
m \ddot{z}
\end{array}\right]=R_{I B}\left[\begin{array}{c}
-T_{1}+T_{3} \\
-T_{2}+T_{4} \\
\sum F_{i}
\end{array}\right]+\left[\begin{array}{l}
0 \\
0 \\
g
\end{array}\right]
$$

where four nozzles are placed as Fig. 2, which generate constant force while it turned on. Since the thruster force vector passes through the center of mass in the quadcopter, it does not affect the rotation motion of the quadcopter and the dynamic equation in rotation is still as same as the conventinal quadcopter.

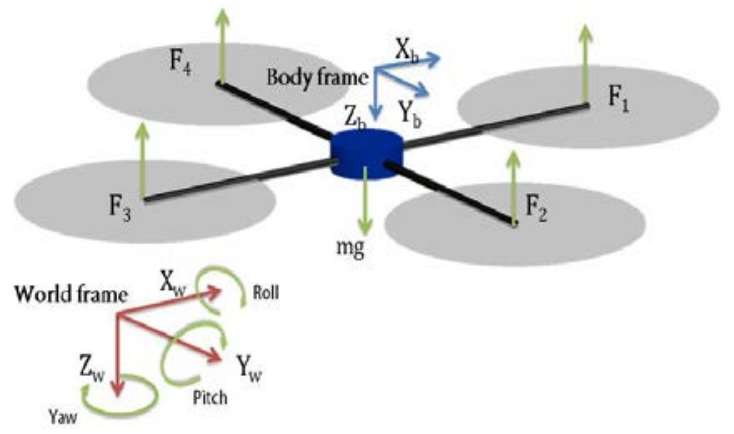

Figure 1. Structural sketch of the coordinate system and forces acting on the coventional quadcopter.

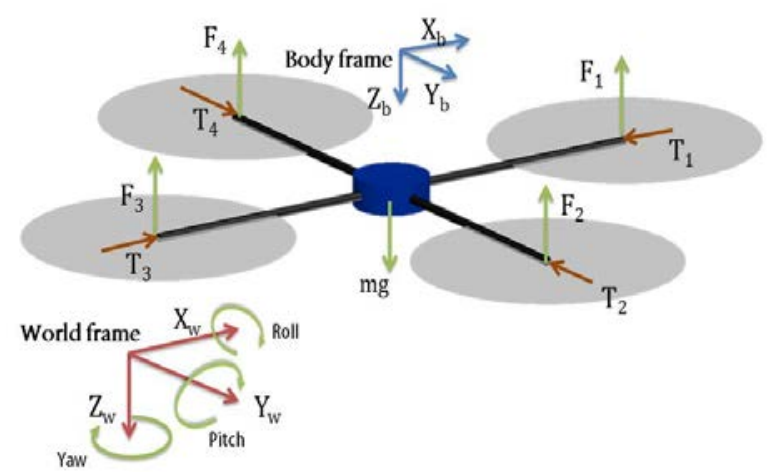

Figure 2. Structural sketch of the coordinate system and forces acting on the quadcopter with additional four jet thrusters.

\section{Controller Design}

This section presents the dual loop state-feedback controller and jet thruster controller used in the proposed quadcopter. Since the quadcopter is known to be an under-actuated system, it cannot control 6 degree-offreedom at the same time. Due to the restrict condition, the controller of the horizontal positioning is separated from the z-axis translation, roll, pitch and yaw 4 degrees of freedom.

In inner loop state feedback controller, the states are chosen as Eq. 2

$$
\begin{gathered}
x_{i}=\left[\begin{array}{llllllll}
z & \dot{z} & \theta & \dot{\theta} & \phi & \dot{\phi} & \psi & \dot{\psi}
\end{array}\right]^{T} \\
\dot{x}_{i}=A_{i} x_{i}+B_{i} u_{i}+\left[\begin{array}{llllllll}
0 & 1 & 0 & 0 & 0 & 0 & 0 & 0
\end{array}\right]^{T} g
\end{gathered}
$$

where

$$
\begin{gathered}
A_{i}=\left[\begin{array}{llllllll}
0 & 1 & 0 & 0 & 0 & 0 & 0 & 0 \\
0 & 0 & 0 & 0 & 0 & 0 & 0 & 0 \\
0 & 0 & 0 & 1 & 0 & 0 & 0 & 0 \\
0 & 0 & 0 & 0 & 0 & 0 & 0 & 0 \\
0 & 0 & 0 & 0 & 0 & 1 & 0 & 0 \\
0 & 0 & 0 & 0 & 0 & 0 & 0 & 0 \\
0 & 0 & 0 & 0 & 0 & 0 & 0 & 1 \\
0 & 0 & 0 & 0 & 0 & 0 & 0 & 0
\end{array}\right], \\
B_{i}=\left[\begin{array}{ccccccc}
0 \frac{-\cos \theta \cos \phi}{m} & 0 & 0 & 0 & -\frac{l}{I_{y y}} & 0 & -\frac{K_{f}}{K_{m}} \frac{1}{I_{z z}} \\
0 \frac{-\cos \theta \cos \phi}{m} & 0 & -\frac{l}{I_{x x}} & 0 & 0 & 0 & \frac{K_{f}}{K_{m}} \frac{1}{I_{z z}} \\
0 \frac{-\cos \theta \cos \phi}{m} & 0 & 0 & 0 & \frac{l}{I_{y y}} & 0 & -\frac{K_{f}}{K_{m}} \frac{1}{I_{z z}} \\
0 \frac{-\cos \theta \cos \phi}{m} & 0 & \frac{l}{I_{x x}} & 0 & 0 & 0 & \frac{K_{f}}{K_{m}} \frac{1}{I_{z z}}
\end{array}\right]^{T} \\
u_{i}=\left[\begin{array}{llllll}
F_{1} & F_{2} & F_{3} & F_{4}
\end{array}\right]^{T}
\end{gathered}
$$

and $B_{i}$ is function of $\theta, \varphi$ and $\phi$ The inner loop controller uses conventional state-feedback, which $u_{i}$ is design to be Eq. 3.

$$
u_{i}=-k_{i} x_{i}-B_{i}^{\dagger}\left[\begin{array}{llllllll}
0 & 1 & 0 & 0 & 0 & 0 & 0 & 0
\end{array}\right]^{T} g
$$

where $B_{i}^{\dagger}$ is Moore-Penrose pseudo inverse of $B_{i}$. The stability of the system is ensured by using a linearquadratic regulator to find a $k_{i}$ that minimizes the cost function by solving the algebraic Riccati equation.

The proposed design for the inner loop controller enables control of rolling and pitching, which means the position of quadcopter can be changed by tilting it and have component force acting on its side. To control the position, an outer loop is designed by using another statefeedback controller, which the state is chosen as Eq. 4.

$$
\begin{gathered}
x_{o}=\left[\begin{array}{cccc}
x & \dot{x} & y & \dot{y}
\end{array}\right]^{T} \\
\dot{x}_{o}=A_{o} x_{o}+B_{o} u_{o}
\end{gathered}
$$


Through the system dynamic equations, the relationships between rolling and pitching angle $\theta$ and $\varphi$ to $x$ and $y$ axis acceleration in inertia frame are

$$
\left[\begin{array}{c}
\dot{x} \\
\ddot{x} \\
\dot{y} \\
\ddot{y}
\end{array}\right]=\frac{\sum F}{m}\left[\begin{array}{c}
0 \\
\sin \psi \sin \phi+\cos \psi \sin \theta \cos \phi \\
0 \\
\sin \psi \sin \theta \cos \phi-\cos \psi \sin \phi
\end{array}\right]
$$

Since $\theta$ and $\varphi$ would be in small angle when the quadcopter is hovering, the equation can be simplified by linearization of the dynamic equations, which can be written as

$$
\left[\begin{array}{c}
\dot{x} \\
\ddot{x} \\
\dot{y} \\
\ddot{y}
\end{array}\right]=\frac{\sum F}{m}\left[\begin{array}{cc}
0 & 0 \\
\cos \psi & \sin \psi \\
0 & 0 \\
\sin \psi & -\cos \psi
\end{array}\right]\left[\begin{array}{l}
\theta \\
\phi
\end{array}\right]
$$

and the dynamic equations Eq. 4 can be written in state space representation, where

$$
\begin{gathered}
A_{o}=\left[\begin{array}{llll}
0 & 1 & 0 & 0 \\
0 & 0 & 0 & 0 \\
0 & 0 & 0 & 1 \\
0 & 0 & 0 & 0
\end{array}\right], \quad B_{o}=\frac{\sum F}{m}\left[\begin{array}{cc}
0 & 0 \\
\cos \psi & \sin \psi \\
0 & 0 \\
\sin \psi & -\cos \psi
\end{array}\right] \\
u_{o}=\left[\begin{array}{ll}
\theta & \phi
\end{array}\right]^{T}=-k_{o} x_{o}
\end{gathered}
$$

and by using same method to determine inner loop controller design, a proper $k_{o}$ can be found.

In this structure, the jet thrusters are considered an auxiliary force that increases positioning accuracy. A variable structure controller design method is used to control jet thrusters. This method is chosen because the jet thruster only has two states, turned on or turned off. And a pair of thrusters can be set to provide force $+T$, $0,-T$ applied on the quadcopter. The phase portrait analysis is used to design the controller and present in following simulation.

Eq. 7 is a system with mass $m$ applied an external force $F$.

$$
\begin{aligned}
& \ddot{x}=\frac{F}{m}=u \\
& \dot{x}=u t+C_{0} \\
& x=\frac{1}{2} u t^{2}+C_{0} t+C_{1}
\end{aligned}
$$

where $C_{0}$ and $C_{1}$ are constants that depend on the initial system state.

Define the variables $x_{1}$ and $x_{2}$

$$
\begin{aligned}
& x_{1}(t)=x(t) \\
& x_{2}(t)=\dot{x}(t)
\end{aligned}
$$

Hence, the phase plane trajectory can be plotted with the relation between $x_{1}$ and $x_{2}$

$$
x_{1}=\frac{1}{2} \frac{m}{F} x_{2}^{2}+\frac{1}{2} \frac{m}{F} C_{0}-\frac{m}{F} C_{0}^{2}+C_{1}
$$

In Fig. 3, the solid lines show the phase trajectories when the positive thruster is turned on, and the dotted lines show the phase trajectories when the negative thruster is turned on.

The next steps are designing the controller and choosing the switching surface. The main concept is when the system state reached the designed switching surface, the states would keep on it by changing $u$. It leads to the origin in any initial state [9]. In Fig. 4, the switching surface $\mathrm{S}$ (Eq. 9) is separated into two structures, region1 and region 2.

$$
S\left(x_{1}, x_{2}\right)= \begin{cases}x_{1}+\frac{1}{2} \frac{m}{F} x_{2}^{2}=0, & \text { when } x_{1}<0 \text { and } x_{2}>0, \\ x_{1}-\frac{1}{2} \frac{m}{F} x_{2}^{2}=0, & \text { when } x_{1}>0 \text { and } x_{2}<0 .\end{cases}
$$

When the system state is in region $1, u$ is set to $-T$ which would lead the state following the dotted trajectory and reach the designed surface. However, $u$ would be set as $T$ and state follows the solid trajectory reaching the surface.

In Fig. 5 shows the overall system block diagram of the controller. The pose controller is designed as a dual loop controller which inner loop controls z-axis, roll, pitch, yaw and outer loop controls $\mathrm{x}$-axis and y-axis. Jet thruster controller is considered as an auxiliary component for higher accuracy positioning.

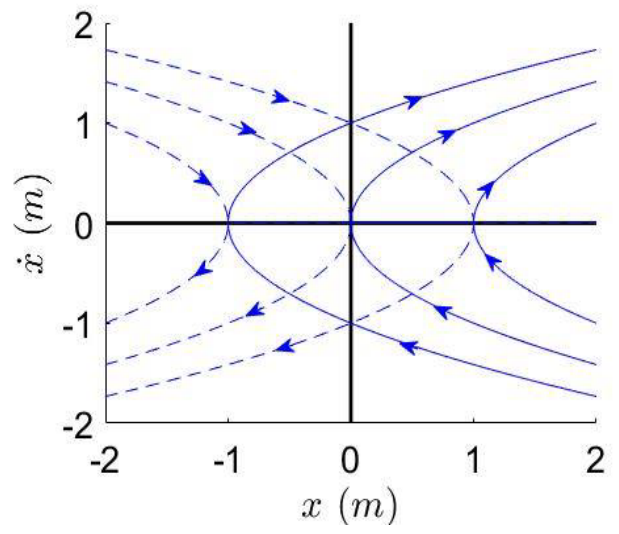

Figure 3. System state trajectory with an external force applied.

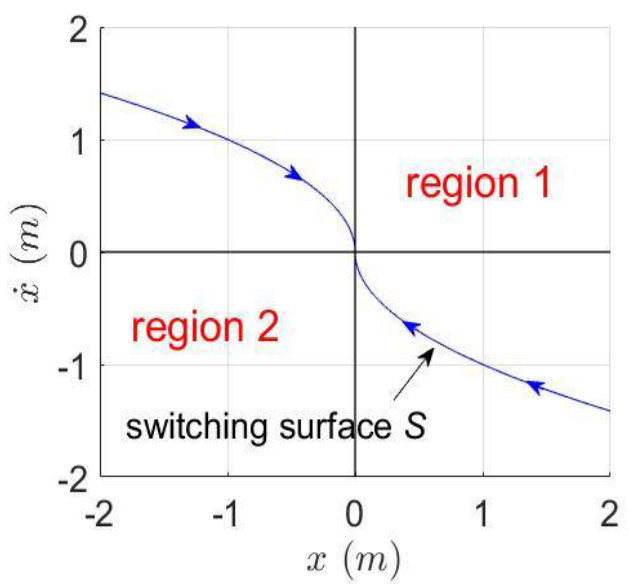

Figure 4. Switching surface $S$ of the jet thruster controller. 


$$
u\left(x_{1}, x_{2}\right)=\left\{\begin{array}{cc}
T, & \text { if }\left(x_{1}, x_{2}\right) \text { in rigion } 1 \\
0, & \text { if }\left(x_{1}, x_{2}\right) \text { on switching surface } \\
-T, & \text { if }\left(x_{1}, x_{2}\right) \text { in rigion } 2
\end{array}\right.
$$

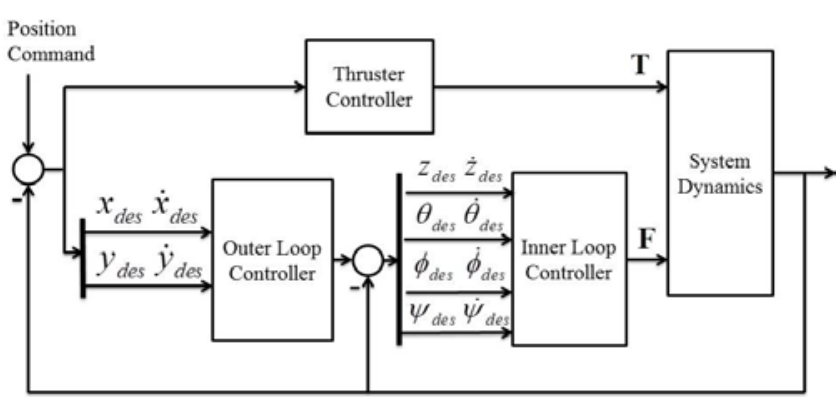

Figure 5. The overall system block diagram of the controller.

\section{Simulation Results}

This section compares simulation results between the proposed quadcopter and a conventional quadcopter that only uses a dual loop state feedback controller and the quadcopter with jet thruster controller is applied. The system equation of motion described in section 3 is implemented in simulations performed by Simulink in MATLAB.

Simulations of two conditions are set up and presented. One is step input response, which is commonly used to evaluate a system performance. Another is testing loiter with external random force applied on the quadcopter to simulate environmental disturbance in order to observe positioning performance and wind rejection.

$$
u\left(x_{1}, x_{2}\right)=\left\{\begin{array}{cl}
u\left(x_{1}, x_{2}\right), & \text { if } x_{1}^{2}+x_{2}^{2} \geq \sigma \\
0, & \text { if } x_{1}^{2}+x_{2}^{2}<\sigma
\end{array}\right.
$$

where $\sigma$ is the threshold that control the deadzone region.

In order to reduce the cost of gas while state near the origin and cause oscillation of jet thruster output, dead zone is added which follows the Eq. 11.

\subsection{Step Input Response Results}

The initial position of the quadcopter is placed at $\{x, y, z\}=\{0,0,0\}$ and set the goal to $\{x, y, z\}=\{1,1,0\}$. Fig.6 shows step response of positioning. It can be observed that quadcopter with thruster have shorter convergence time compare to conventional one and have less overshoot. Fig.7 shows that the phase trajectory follows the designed switching surface to the setting goal directly without having spiral phase trajectory. Fig.8 shows the output of nozzles on $\mathrm{x}$ and $\mathrm{y}$ axis.

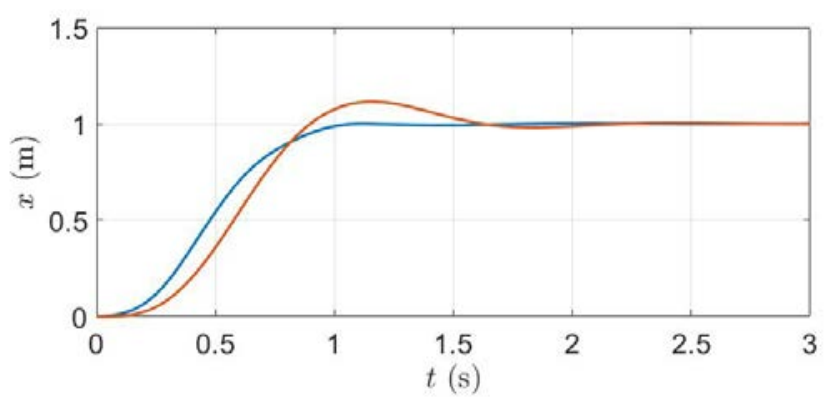

(a)

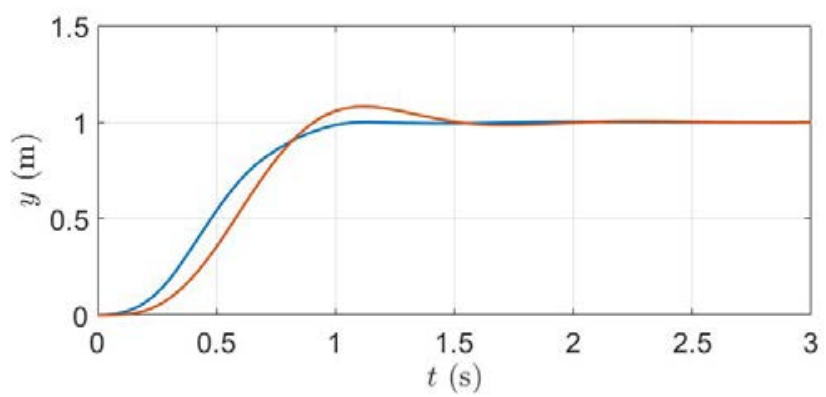

(b)

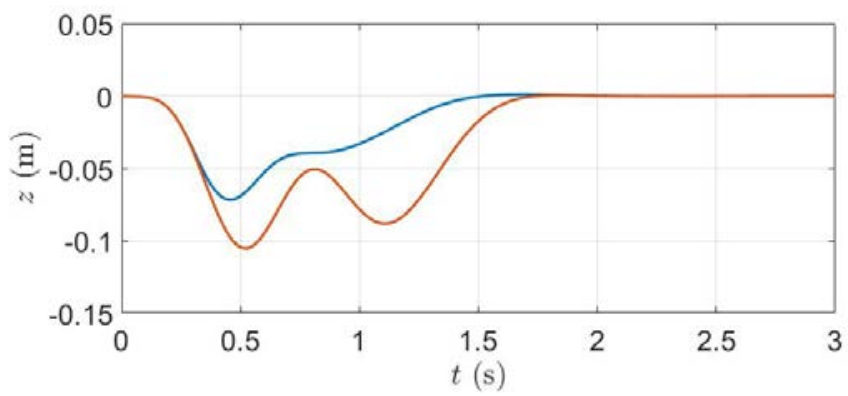

(c)

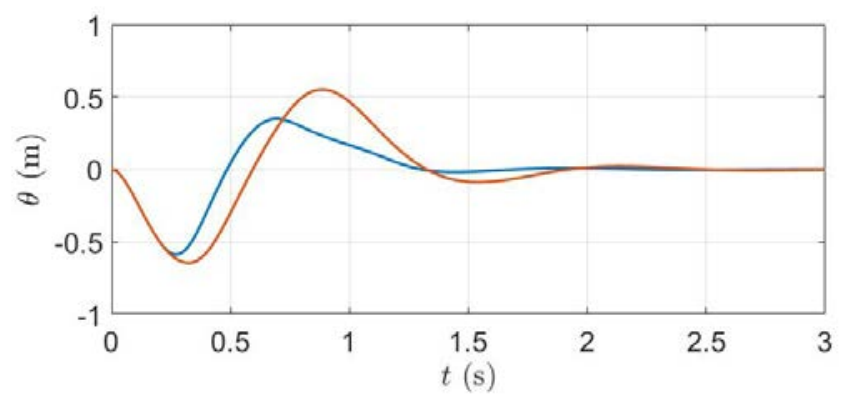

(d)

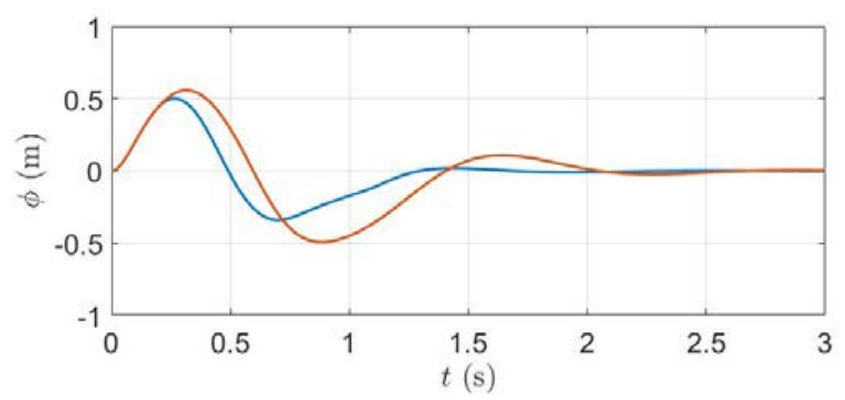

(e) 


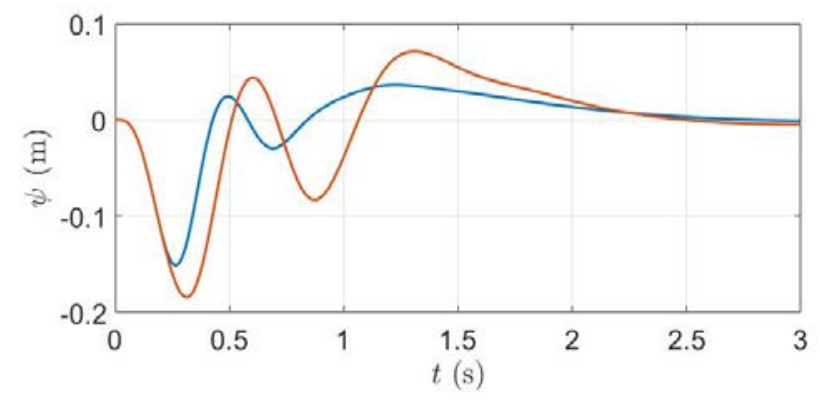

(f)

\section{— with jet thruster — without jet thruster}

Figure 6. Simulation of system response to a step input.(a)(b)(c) translation motion, $(\mathrm{d})(\mathrm{e})(\mathrm{f})$ rotation motion.

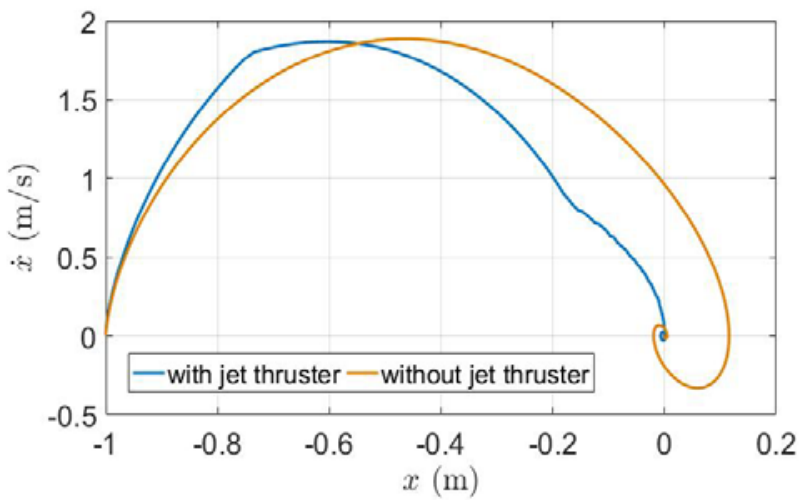

(a)

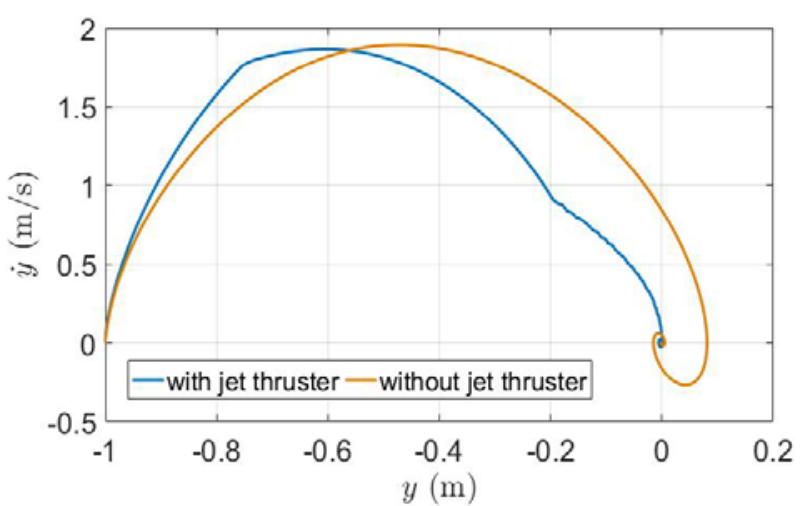

(b)

Figure 7. State error trajectory on (a) x-axis (b) y-axis.

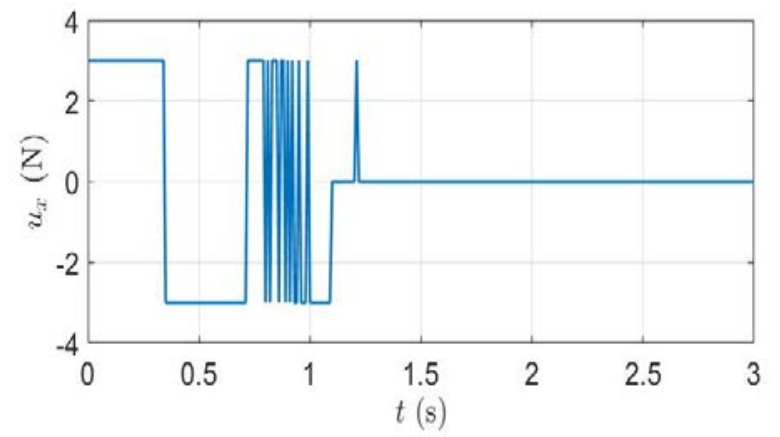

(a)

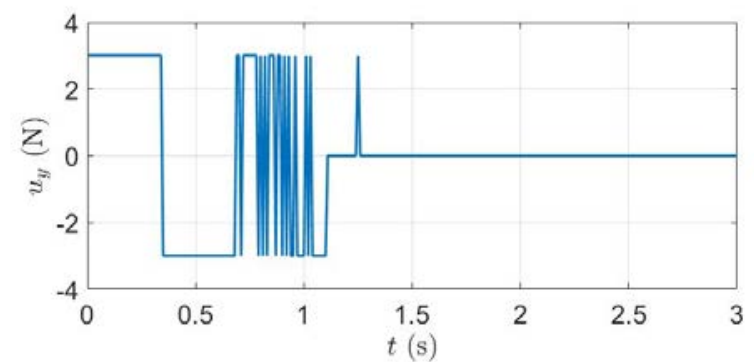

(b)

Figure 8. Control input of $3 \mathrm{~N}$ jet thruster (a) $\mathrm{x}$-axis (b) $\mathrm{y}$-axis.

\subsection{Loiter Results.}

The high accuracy positioning performance of the jet thruster controller was tested by simulating loiter in a disturbed environment. In Fig. 9 shows that the quadcopter equipped with jet thrusters resisted external force over 10s. To simulate the wind disturbance, a random force within maxima $5 \mathrm{~N}$ is applied on the $\mathrm{x}$-axis and $\mathrm{y}$-axis of quadcopter. A conventional quadcopter requires a large pitch angle when the external force is high in the horizontal direction. It cost longer time to create enough resist force than by adding a thruster on its side. Table.1 summarizes the RMS error of the quadcopter. The simulation results show that the quadcopter equipped with jet thruster can have better positioning than the conventional one which decreases about $85 \%$ of RMS error.

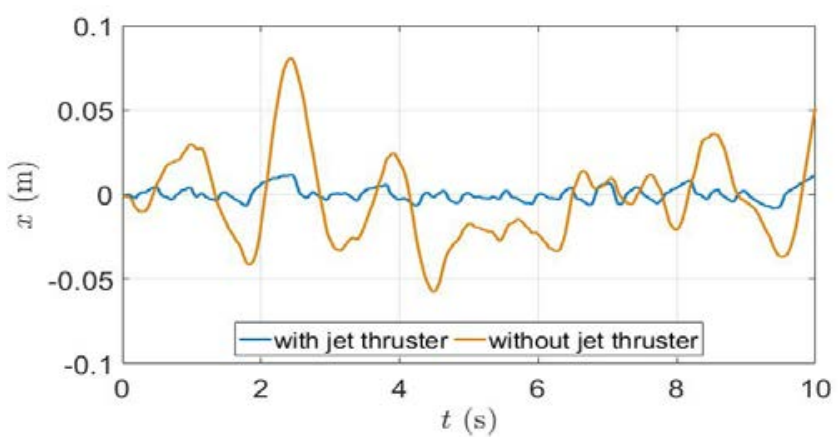

(a)

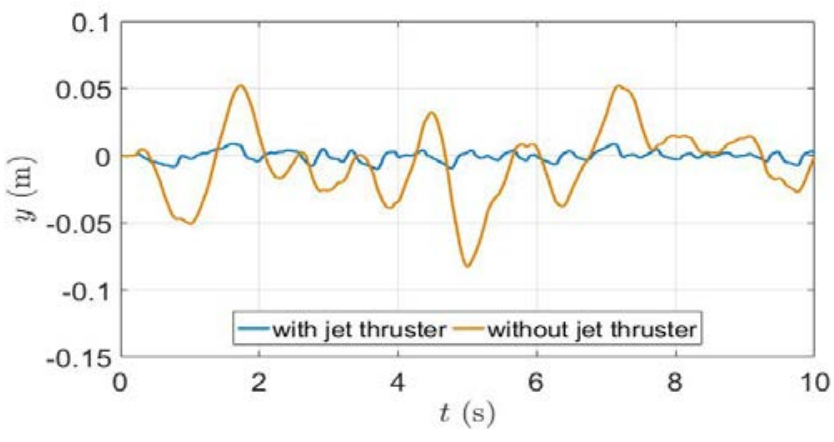

(b) 


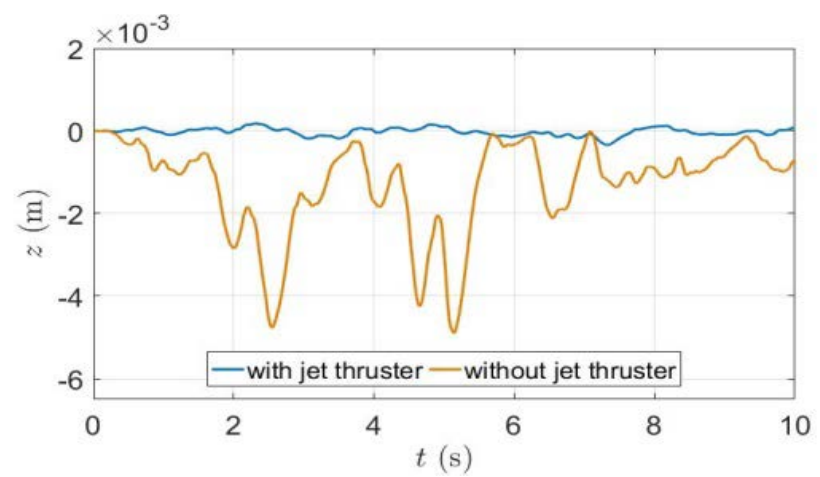

(c)

Figure 9. Simulation of positioning performance with disturbance over 10s. (a) x-axis (b) y-axis (c) z-axis.

Table 1. RMS error over 10s loiter simulation under external force shown in Fig. 9.

\begin{tabular}{|c|c|c|c|}
\hline & $\begin{array}{c}e_{x} \text { RMS } \\
(\mathrm{cm})\end{array}$ & $\begin{array}{c}e_{y} \text { RMS } \\
(\mathrm{cm})\end{array}$ & $\begin{array}{c}e_{\mathrm{z}} \mathrm{RMS} \\
(\mathrm{cm})\end{array}$ \\
\hline $\begin{array}{c}\text { Traditional } \\
\text { quadcopter }\end{array}$ & 2.69 & 2.78 & 0.17 \\
\hline $\begin{array}{c}\text { Quadcopter } \\
\text { equipped with } \\
\text { jet thruster }\end{array}$ & 0.41 & 0.38 & 0.01 \\
\hline
\end{tabular}

\section{Conclusions}

To investigate the control problem of an over actuated system, a quadcopter model with simple dual loop state feedback controller with the auxiliary jet thrusters is created and simulated. Based on the dynamic equations for a second order system, the jet thruster controller is designed to lead the phase trajectory of state error to the origin. In the simulation result it shows that it has better performance compared with the conventional system. The horizontal RMS error in loiter simulation can decrease about $85 \%$. However, keeping the state on the designed trajectory requires excessive energy due to cancel out the force generated from the simple outer loop of the state feedback.

In future works, we will improve the dual loop controller which enter the switching surface smoother and keep tracking on it. Further studies are also needed to analyze how the switching frequency of the thruster affects fuel consumption. Further hardware tests are also needed.

\section{References}

1. James R. Kutia, Karl A. Stol and Weiliang $\mathrm{Xu}$, Canopy sampling Using an Aerial Manipulator: A Pre-liminary Study (ICUAS, Denver, Colorado, USA, Jun. 9-12, 2015)

2. J.X.J. Bannwarth, Z. J. Chen, K.A.Stol, Disturbance Accomodation Control for Wind, International Conference on Unmanned Aircraft System(ICAUAS, Arlington, VA, USA, June 7-10, 2016)

3. Chau T. Ton and Willam Mackunis, Robust Attitude Tracking Control of a Quadrotor Helicopter in the Presence of Uncertainty, (51st IEEE Conference on Decision and Control, Maui, Hawaii, USA, Dec. 1013, 2012)

4. Pang Tao, DESIGN, PROTOTYPING AND AUTONOMOUS CONTROL OF GASOLINEENGINE VARIABLE-PITCH QUADCOPTER, Electical and computer engineering national university of singapore (2016)

5. Chin E. Lin, Thanakorn Supsukbaworn, Development of Dual Power Multirotor System, International Journal of Aerospace Engineering Volume 2017, ID 9821401, 19 pages (2017)

6. John G. Furumo, COLD-GAS PROPULSION FOR SMALL SATELLITE ATTITUDE CONTROL, STATION KEEPING, AND DEORBIT, Hawai'i Space Grant Consortium Undergraduate Fellowship Reports, HSGC Report No. 13-22. Hawai'i Space Grant Consortium, Honolulu. 67-78. (2013)

7. Assad Anis, Design \& Development of Cold Gas Propulsion System for Pakistan Remote Sensing Satellite (PRSS), (2nd ICAST, Islamabad, Pakistan, 29th - 30th Nov. 2008)

8. Sarah L. Nothnagel, Professor Jeffrey A. Hoffman and Brett J. Streetman, Development of a Cold Gas Propulsion System for the TALARIS Hopper, Science in Aeronautics and Astronautics, Massachusetts Institute of Technology. Retrieved from http://ssl.mit.edu/publications/theses/SM-2011NothnagelSarah.pdf (2011)

9. Michal Athans: Minimum-Fuel Feedback Control Systems: Second-Order Case, IEEE Trans. Appl. Ind., vol. 82, no. 65, p. 8-17, Mar. (1963). 